Virginia Commonwealth University VCU Scholars Compass

2010

\title{
Electronic and magnetic properties of a $\mathrm{BN}$ sheet decorated with hydrogen and fluorine
}

Jian Zhou

Peking University

Qian Wang

Virginia Commonwealth University

Qiang Sun

Peking University, Virginia Commonwealth University

Puru Jena

Virginia Commonwealth University, pjena@vcu.edu

Follow this and additional works at: http://scholarscompass.vcu.edu/phys_pubs

Part of the Physics Commons

Zhou, J., Wang, Q. Sun, Q. et al. Electronic and magnetic properties of a BN sheet decorated with hydrogen and fluorine. Physical Review B, 81, 085442 (2010). Copyright (C) 2010 American Physical Society.

\section{Downloaded from}

http://scholarscompass.vcu.edu/phys_pubs/67

This Article is brought to you for free and open access by the Dept. of Physics at VCU Scholars Compass. It has been accepted for inclusion in Physics Publications by an authorized administrator of VCU Scholars Compass. For more information, please contact libcompass@vcu.edu. 


\title{
Electronic and magnetic properties of a BN sheet decorated with hydrogen and fluorine
}

\author{
Jian Zhou, ${ }^{1}$ Qian Wang, ${ }^{2}$ Qiang Sun, $,{ }^{1,2}, *$ and Puru Jena ${ }^{2}$ \\ ${ }^{1}$ Department of Advanced Materials and Nanotechnology and Center for Applied Physics and Technology, \\ Peking University, Beijing 100871, China \\ ${ }^{2}$ Department of Physics, Virginia Commonwealth University, Richmond, Virginia 23284, USA \\ (Received 28 October 2009; revised manuscript received 7 January 2010; published 25 February 2010)
}

\begin{abstract}
First-principles calculations based on density-functional theory reveal some unusual properties of BN sheet functionalized with hydrogen and fluorine. These properties differ from those of similarly functionalized graphene even though both share the same honeycomb structure. (1) Unlike graphene which undergoes a metal to insulator transition when fully hydrogenated, the band gap of the BN sheet significantly narrows when fully saturated with hydrogen. Furthermore, the band gap of the BN sheet can be tuned from 4.7 to $0.6 \mathrm{eV}$ and the system can be a direct or an indirect semiconductor or even a half-metal depending on surface coverage. (2) Unlike graphene, the hydrogenation of BN sheet is endothermic. (3) Unlike graphene, BN sheet has heteroatomic composition. When codecorated with $\mathrm{H}$ and $\mathrm{F}$, it can lead to anisotropic structures with rich electronic and magnetic properties. (4) Unlike graphene, BN sheets can be made ferromagnetic, antiferromagnetic, or magnetically degenerate depending on how the surface is functionalized. (5) The stability of magnetic coupling of functionalized $\mathrm{BN}$ sheet can be further modulated by applying external strain. Our study highlights the potential of functionalized BN sheets for unusual applications.
\end{abstract}

DOI: 10.1103/PhysRevB.81.085442

PACS number(s): $36.40 . \mathrm{Cg}$

\section{INTRODUCTION}

Dimensionality has become an important parameter to tune the properties of materials. Currently, tremendous effort has been devoted to two-dimensional (2D) atomic-layerbased materials. One of such example is graphene ${ }^{1}$ which has many advantages compared to carbon nanotubes (CNTs) when used in polymeric composites. The covalently bonded honeycomb lattice of the graphene sheet shows high stability and exceptional properties ${ }^{2}$ such as high electron mobility even at room temperature, ambipolar effect, Klein tunneling, and anomalous quantum Hall effect. Especially, electrons in graphene sheet behave like mass-less Dirac fermions making the observation of several relativistic effects possible. The impressive progress in graphene research has motivated scientists to explore other 2D atomic based materials. Among them, BN sheet has become a hotly pursued system as it shares the same honeycomb lattice structure as graphene. Using a chemical-solution-derived method starting from single-crystalline hexagonal boron nitride, Han and coworkers successfully synthesized $\mathrm{BN}$ monoatomic layer. ${ }^{3}$ Very recently, an efficient method to fabricate high-yield 2D BN sheets has been developed by using a sonicationcentrifugation technique ${ }^{4}$ where milligram quantity of $\mathrm{BN}$ sheets are achievable and ultimately pure BN sheets can be obtained based on a highly pure precursor. The sheet thickness can be adjusted by the centrifugation speed.

The advances in experimental synthesis of $\mathrm{BN}$ sheet have led us to explore the properties of $\mathrm{BN}$ sheet by decorating its surface. Although BN sheet has similar geometry as graphene sheet, it can display different properties. For example, unlike graphite, BN layers are stable under high temperature up to $1000 \mathrm{~K}$. BN sheet is semiconducting while graphene sheet is metallic. In order to open a band gap in graphene, surface modification such as hydrogenation is needed. The resulting graphane sheet ${ }^{5,6}$ has a band gap of 4.5
$\mathrm{eV}$ which is tunable by modifying its surface with atoms such as F. ${ }^{7}$ Similar decoration of BN sheet raises some interesting questions: (1) since the BN sheet is already semiconducting with a band gap of $4.7 \mathrm{eV}$, how would its band gap change when hydrogenated or fluorinated? (2) When half of the $\mathrm{H}$ atoms are removed from a fully hydrogenated graphene sheet (graphane), the resulting semihydrogenated graphene sheet (graphone) becomes ferromagnetic, ${ }^{8}$ but it is still nonmagnetic when semifluorinated. What would be the magnetic properties of semihydrogenated or semifluorinated $\mathrm{BN}$ sheet? Is it ferromagnetic, antiferromagnetic, or nonmagnetic? (3) All C atoms in graphene sheet are equivalent with covalent bonding between them. In contrast, $\mathrm{B}$ and $\mathrm{N}$ sites in $\mathrm{BN}$ sheet are not equivalent. The charge transfer from $\mathrm{B}$ to $\mathrm{N}$ permits the bonding between them to be more ionic, thus the properties of semidecorated $\mathrm{BN}$ sheet will depend on which sites are decorated with $\mathrm{H}$ and/or F. How would codecoration of $\mathrm{BN}$ sheet with $\mathrm{H}$ on one side and $\mathrm{F}$ on the other side affect its electronic and magnetic properties?

In this paper, we have made extensive studies of the electronic structure and magnetic properties of fully decorated and semidecorated $\mathrm{BN}$ sheets using $\mathrm{H}$ and/or $\mathrm{F}$ atoms. We show that for a fully hydrogenated BN sheet, the energy band gap is reduced from that in pristine $\mathrm{BN}$ sheet. With semidecoration on different sites, BN sheet can display ferromagnetic $(\mathrm{FM})$ or antiferromagnetic $(\mathrm{AF})$ properties. And for semifluorinated $\mathrm{BN}$ sheet, contrary to what has been found in the case of $\mathrm{BN}$ nanotube, $\mathrm{FM}$ and $\mathrm{AF}$ states are nearly degenerated. In addition, the relative stability of FM and AF states can be further modulated by applying external strain.

\section{METHODS}

Our calculations are based on spin-polarized densityfunctional theory (DFT) using generalized gradient approxi- 


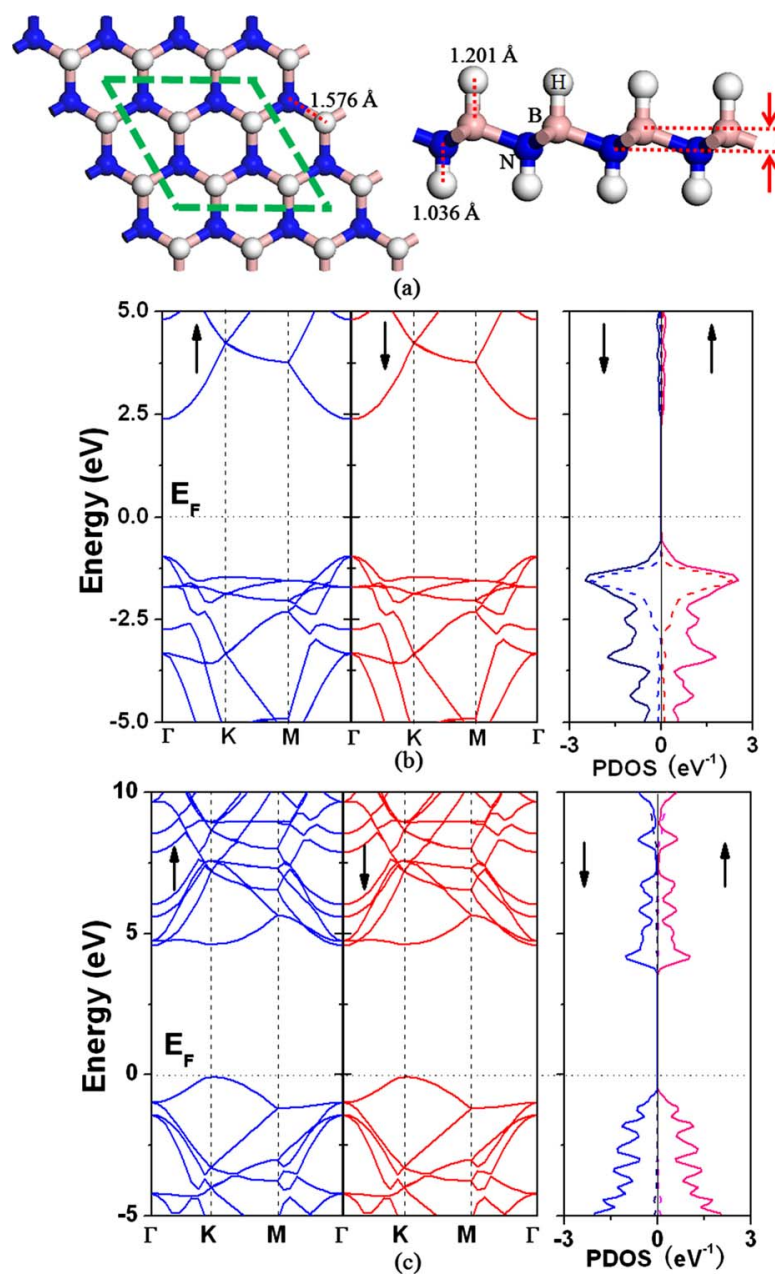

FIG. 1. (Color online) (a) Optimized structure of hydrogenated $\mathrm{BN}$ nanosheet, H-BN-H. The dashed rhombus outlines the supercell used in our calculation. (b) and (c) are calculated band structure and PDOS of $\mathrm{H}-\mathrm{BN}-\mathrm{H}$ and pristine BN sheet, respectively. In PDOS, dashed and solid curves correspond to $s$ and $p$ orbitals, respectively.

mation (GGA) (Ref. 9) for exchange-correlation potential. We have used Perdew-Burke-Ernzerhof (PBE) functional for GGA as implemented in the Vienna ab initio Simulation Package (VASP). ${ }^{10,11}$ For the geometric and electronic structural calculations, a supercell consisting of fourfold unit cells of $h$-BN sheet is used with a vacuum space of $15 \AA$ between two layers to avoid interactions between them. Pseudopotentials with $2 s^{2} 2 p^{1}, 2 s^{2} 2 p^{3}, 1 s^{1}$, and $2 s^{2} 2 p^{5}$ valence electron configurations, respectively, for $\mathrm{B}, \mathrm{N}, \mathrm{H}$, and $\mathrm{F}$ atoms are used. The Brillouin zone is represented by Monkhorst-Pack special $k$-point mesh ${ }^{12}$ of $7 \times 7 \times 1$. The energy cutoffs, convergence in energy, and force are set to $400 \mathrm{eV}, 1$ $\times 10^{-4} \mathrm{eV}$, and $0.01 \mathrm{eV} / \AA$, respectively. Optimizations are performed using conjugated gradient method and without any symmetric constraints. The accuracy of our calculation procedure is tested using pristine $h$-BN sheet. The optimized bond length of B-N of $1.446 \AA$ is in good agreement with experimental value of $1.45 \AA$. The calculated band gap of $\mathrm{BN}$ sheet of $4.71 \mathrm{eV}$ (Fig. 1) is also in good agreement with previous theoretical result. ${ }^{13}$

\section{RESULTS AND DISCUSSION}

In the following, we discuss the electronic structure and properties of BN sheet when (1) fully hydrogenated, (2) semihydrogenated, (3) semifluorinated, (4) under influence of external strain, and (5) semihydrogenated and semifluorinated.

\section{A. Fully hydrogenated BN sheet}

Following the idea of hydrogenation of graphene sheet, we first discuss the results for a fully hydrogenated $h$-BN sheet (labeled as H-BN-H) which is very similar in geometry to its carbon counterpart, graphane, as shown in Fig. 1(a), where $\mathrm{B}$ and $\mathrm{N}$ atoms become $s p^{3}$ hybridized which distorts the planar geometry forming a zigzag configuration as $\mathrm{C}$ atoms do in graphane. ${ }^{6}$ The distance between $\mathrm{B}$ and $\mathrm{N}$ planes is found to be $0.518 \AA$. The hydrogen atoms are adsorbed on the top site of $\mathrm{B}$ and $\mathrm{N}$ atoms with $\mathrm{H}-\mathrm{B}$ and $\mathrm{H}-\mathrm{N}$ bond lengths of 1.201 and $1.036 \AA$, respectively. The bond length of $\mathrm{H}-\mathrm{B}$ is a little larger than that of H-N bond length because of the difference in bonding. Charge analysis with WignerSeitz partition suggests that $\mathrm{H}$ atoms adsorbed on $\mathrm{B}$ and $\mathrm{N}$ are both positively charged carrying charges of 0.012 and 0.129 electrons, respectively. To study the energetics of hydrogenation, we calculated the formation energy which is defined as the energy difference between the hydrogenated $\mathrm{BN}$ sheet, pristine $\mathrm{BN}$ sheet, and $\mathrm{H}_{2}$ molecules. We found that, in contrast to the graphene sheet, the hydrogenation of $\mathrm{BN}$ sheet is endothermic with energy of $0.195 \mathrm{eV} / \mathrm{H}$.

The energy-band structure and partial density of state (PDOS) of H-BN-H are plotted in Fig. 1(b). It is found to be nonmagnetic with a direct band gap of $3.33 \mathrm{eV}$, which is lower than that in the pristine BN sheet, namely, $4.71 \mathrm{eV}$. The valence-band maximum (VBM) and conduction-band minimum $(\mathrm{CBM})$ are both located at the $\Gamma$ point in the reciprocal space. Here we see some differences between fully hydrogenated graphene and $\mathrm{BN}$ sheet. Hydrogenation opens a band gap in the former while it reduces the band gap in the latter. In fact, introduction of two $\mathrm{H}$ atoms introduces two occupied energy bands (one in majority and one in minority band sharing the same energy). These are located just above the valence band of pristine BN sheet. Accordingly, the band gap of the system is reduced.

\section{B. Semihydrogenated BN sheet}

As stated in previous discussions, the hydrogenation of $\mathrm{BN}$ sheet is an endothermic process. Once fully hydrogenated, $\mathrm{H}$ can bind on $\mathrm{B}$ and $\mathrm{N}$ sites with the bond lengths of 1.201 and $1.036 \AA$, respectively, which are comparable to the bond lengths of 1.23 and $1.03 \AA$ for the corresponding $\mathrm{BH}$ and $\mathrm{NH}$ dimers in free states. ${ }^{14}$ This suggests that $\mathrm{H}$ can strongly bind to the distorted nonplanar BN sheet. This can be understood by considering the fact that distortion changes the bonding between $\mathrm{B}$ and $\mathrm{N}$ and enhances the reactivity. Now the next question is: starting from the fully hydrogenated BN sheet, can one find a way to remove hydrogen from one side forming a semihydrogenated $\mathrm{BN}$ sheet? If so, how will the properties change? 


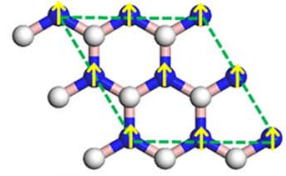

$\mathrm{FM}, \mathrm{m}=4 \mu_{\mathrm{B}}$ $\mathrm{E}=0.000 \mathrm{eV}$
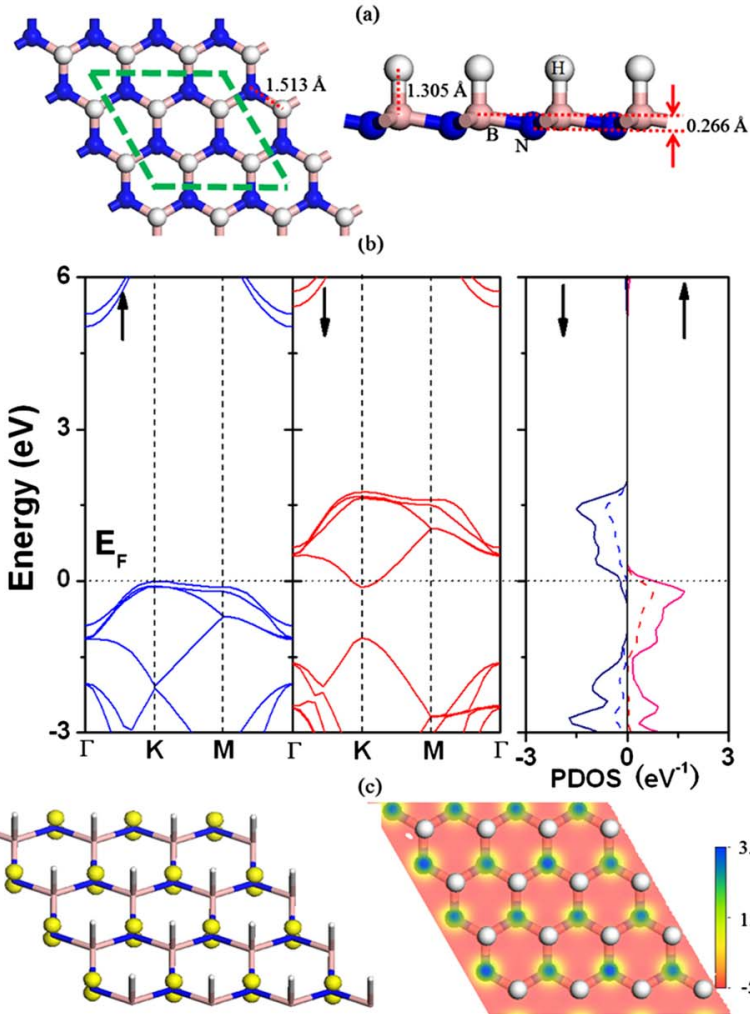

(d)

FIG. 2. (Color online) Calculated results of H-BN. (a) Three magnetic coupling states. (b) Optimized structure of the FM state. (c) Calculated band structure and PDOS of H-BN in FM state. (d) Isosurface $\left(0.5 e / \AA^{3}\right)$ and $2 \mathrm{D}$ slice of spin density $\left(\rho_{\alpha}-\rho_{\beta}\right)$ in FM state.

In graphene sheet, all the $\mathrm{C}$ sites are equivalent. Thus, when removing half of $\mathrm{H}$ atoms from a fully hydrogenated graphene sheet (graphane), we have only one option for the semihydrogenated sheet. ${ }^{8}$ However, in $\mathrm{BN}$ sheet, $\mathrm{B}$ and $\mathrm{N}$ sites are not equivalent and semihydrogenation can be accomplished by removing $\mathrm{H}$ from either $\mathrm{B}$ sites or $\mathrm{N}$ sites. Which is the preferred configuration? To find this, we first considered semihydrogenation by placing $\mathrm{H}$ on $\mathrm{B}$ sites, as shown in Fig. 2. We label this case as H-BN.

The geometric optimization showed that when hydrogen atoms on $\mathrm{N}$ sites are removed, the $\mathrm{BN}$ surface became less distorted and the distance between $\mathrm{B}$ plane and $\mathrm{N}$ plane is reduced to $0.266 \AA$. The H-B bond length is elongated to $1.305 \AA$, which is still comparable to the bond length of $1.23 \AA$ of $\mathrm{BH}$ dimer in a free state. ${ }^{14}$ These results are similar to those found in graphane and graphone. ${ }^{8}$ In H-BN configuration, we found that the $\mathrm{N}$ atoms possess about $1 \mu_{B}$ magnetic moment, while $\mathrm{B}$ and $\mathrm{H}$ atoms carry very small magnetic moments. To verify that the system is indeed magnetic, we calculated the total energies for three magnetic cou- pling configurations between $\mathrm{N}$ atoms [Fig. 2(a)]: (1) FM coupling, (2) AF coupling, and (3) nonmagnetic (NM) coupling. The results showed that the FM coupling between $\mathrm{N}$ atoms is energetically lower than that in AF and NM configurations by 0.153 and $0.158 \mathrm{eV}$, respectively. Since our calculated supercell is based on four unit cells of BN sheet, the energy differences are thus 38 and $40 \mathrm{meV}$ per unit cell, respectively. This indicates that the semihydrogenated $\mathrm{H}-\mathrm{BN}$ nanostructure exhibits FM coupling. The physics involved can be described as the following: in pristine BN sheet, the charge transfer from $\mathrm{B}$ to $\mathrm{N}$ and the orbital hybridization make electrons paired and the system is nonmagnetic. The $2 p_{z}$ electrons of $\mathrm{N}$ atoms contribute to the highest occupied VBM while $2 p_{z}$ electrons of $\mathrm{B}$ atoms contribute to lowest unoccupied CBM. When semihydrogenated on B sites, B atoms are covalently bonded with $\mathrm{H}$ atoms forming $s p^{3}$ hybridization and almost no charge transfer occurs from $\mathrm{B}$ to $\mathrm{N}$. Thus $2 p_{z}$ electrons on $\mathrm{N}$ atoms remain unpaired. The extended $p$ - $p$ interaction results in a long-range magnetic coupling between $2 p$ moments as found in graphone sheet. ${ }^{8}$ Conventionally, magnetic moment comes from unfilled $d$ or $f$ orbitals of metallic atoms. Using mean-field theory and the energy difference between FM and AF states, we can estimate the Curie temperature of $\mathrm{H}-\mathrm{BN}$ by the formula $\gamma k_{B} T_{c} / 2=E_{\mathrm{AF}}-E_{\mathrm{FM}}$, where $\gamma$ is the dimension of the system and $k_{B}$ is Boltzmann constant. ${ }^{15}$ Our estimated results are 293 and $440 \mathrm{~K}$, depending on whether the system is treated as three dimensional (3D) and 2D, respectively.

Band structure and PDOS are plotted in Fig. 2(c). We find that the system is half metallic where majority-spin state remains as an indirect band-gap insulator with band gap of $5.05 \mathrm{eV}$, while minority-spin state is metallic. To visualize the magnetic property clearly, we plotted the isosurface and a two-dimensional slice of spin density [Fig. 2(d)]. We see that the magnetism on $\mathrm{N}$ atoms is mainly contributed by unsaturated $2 p_{z}$ orbital, which is consistent with the results obtained from PDOS. We find that one $\mathrm{H}$ atom adsorbed on $\mathrm{B}$ site introduces one occupied energy band in majority channel as well as one unoccupied energy band with slightly higher energy in the minority channel. The two energy bands are located slightly above the highest occupied valence band of pristine $\mathrm{BN}$ sheet. If the concentration of $\mathrm{H}$ atoms is increased to the level of semihydrogenation, the new induced occupied and unoccupied energy bands will be broadened due to hybridization effect. They will collapse with each other and give rise to half-metallic band structure.

For the semihydrogenation on $\mathrm{N}$ sites (denoted as $\mathrm{H}-\mathrm{NB}$ ), the optimized $\mathrm{H}-\mathrm{NB}$ sheet shows that the distance between $\mathrm{B}$ and N planes is $0.450 \AA$, which is larger than that of $\mathrm{H}-\mathrm{BN}$ case $(0.266 \AA)$ and smaller than that of $\mathrm{H}-\mathrm{BN}-\mathrm{H}$ case $(0.518 \AA)$. The H-N bond length of $1.083 \AA$ is comparable to the bond length of $1.03 \AA$ in $\mathrm{NH}$ dimer in a free state. ${ }^{14}$ Since $\mathrm{B}$ atom has a valence configuration of $2 s^{2} 2 p^{1}$, once $\mathrm{N}$ sites are terminated with $\mathrm{H}$, the charge transfer from $\mathrm{B}$ to $\mathrm{N}$ is diminished, leaving $2 p$ electron spin in B site unpaired. In fact, we found that the unhydrogenated B atoms carried a magnetic moment of about $0.75 \mu_{B}$, while very small net magnetic moment was found on $\mathrm{H}$ or $\mathrm{N}$ atoms. We computed total energies for three magnetic configurations (FM, AF, and $\mathrm{NM})$. The AF state is found to be energetically most stable. 

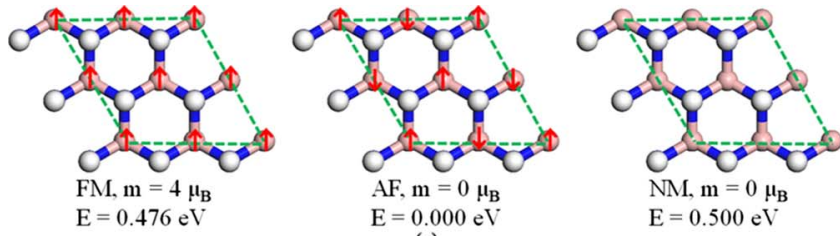

(a)

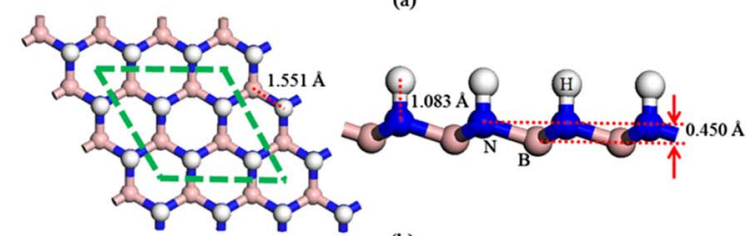

(b)
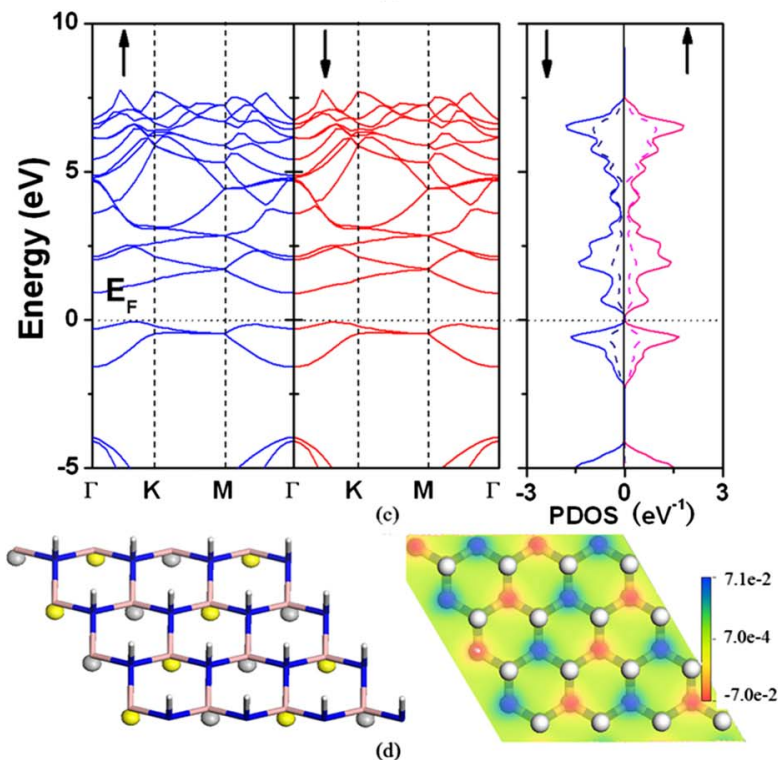

FIG. 3. (Color online) The legend is similar to that in Fig. 2 while results in (b)-(d) are for the AF state. (d) Yellow and gray indicate the positive and negative values, respectively, in the isosurface $\left(0.38 e / \AA^{3}\right)$.

The FM and NM states are higher in energy than that of AF state by 0.476 and $0.500 \mathrm{eV}$, respectively, corresponding to 119 and $125 \mathrm{meV}$ per unit cell. These energy differences include magnetic contribution from both exchange interaction as well as from changes in geometry. We found that in FM state, the H-N bond length is $1.071 \AA$, which is shorter than that in the AF state. The energy of the H-NB in AF state is higher than its isomer $\mathrm{H}-\mathrm{BN}$ in its FM state by $1.36 \mathrm{eV}$, suggesting that $\mathrm{H}-\mathrm{NB}$ configuration is energetically less stable than $\mathrm{H}-\mathrm{BN}$ configuration.

The band structure and PDOS for the AF state of H-NB are plotted in Fig. 3(c), showing that the system is an indirect band-gap semiconductor where band gap is reduced to 0.97 eV. Detailed analysis of PDOS reveals again that the magnetic moment on $\mathrm{B}$ atoms is due to the unsaturated $2 p$ orbital, which is clearly shown in Fig. 3(d). The system with $\mathrm{H}$ atoms adsorbed on $\mathrm{N}$ atoms of $\mathrm{BN}$ sheet in $\mathrm{AF}$ state also shows that some occupied (majority) and unoccupied (minority) energy bands are newly formed just under the conduction band (related to the electron occupation in the lowest unoccupied conduction band arising from B $2 p$ orbital of pristine $\mathrm{BN}$ sheet), which makes the system an $n$-type semiconductor.

\section{Semifluorinated BN sheet}

We see from above that the electronic and magnetic properties of $\mathrm{BN}$ sheet can be tuned by decorating $\mathrm{B}$ or $\mathrm{N}$ sites with $\mathrm{H}$ atoms. In this section, we study the fluorination of BN sheet. The motivations are twofold: (1) studies have already been carried out experimentally and theoretically on fluorination of graphene, ${ }^{7,16} \mathrm{CNT},{ }^{17-19}$ and fullerene $\left[\mathrm{C}_{60} \mathrm{~F}_{18},{ }^{20,21} \mathrm{C}_{60} \mathrm{~F}_{36},{ }^{22,23} \mathrm{C}_{60} \mathrm{~F}_{48},{ }^{23-25} \mathrm{C}_{60} \mathrm{~F}_{60},{ }^{26} \mathrm{C}_{58} \mathrm{~F}_{18}\right.$ (Ref. 27)]. It has been found that fluorination can be used to change CNT from metallic to semiconducting ${ }^{10}$ or from nonmagnetic to magnetic. ${ }^{28}$ Therefore, it is highly desirable to see if fluorination of $\mathrm{BN}$ sheet can induce some new effects. (2) Hydrogenation of $\mathrm{BN}$ sheet is found to be endothermic. One of the reasons is that the bonding in $\mathrm{H}_{2}$ is strong. Since bonding in $\mathrm{F}_{2}$ is weaker than in $\mathrm{H}_{2}$, an interesting question arises: is fluorination of $\mathrm{BN}$ sheet endothermic or exothermic? In this section, we discuss the effect of fluorination of $\mathrm{BN}$ sheet on electronic and magnetic properties.

Because of weak binding between $\mathrm{F}$ and $\mathrm{N}$ atoms, ${ }^{29}$ we only consider semifluorination of $\mathrm{BN}$ sheet in which $\mathrm{F}$ atoms are attached to $\mathrm{B}$ atoms in $\mathrm{BN}$ (termed as F-BN) (Fig. 4). This structure may be synthesized in fluorine plasma environment by protecting one side of $\mathrm{BN}$ sheet. In the optimized geometric structures, the distance between $\mathrm{B}$ and $\mathrm{N}$ planes is found to be $0.375 \AA$, and F-B bond length is $1.415 \AA$, which is a little bit larger than the BF dimer bond length of $1.263 \AA$ in a free state. ${ }^{14}$ In contrast to that found in hydrogenation, fluorination is found to be exothermic and the formation energy is $0.645 \mathrm{eV} / \mathrm{F}$. Again, we found that the $\mathrm{N}$ atoms carry a magnetic moment of about $1 \mu_{B}$. Three magnetic states with their corresponding energies are given in Fig. 4(a), showing that the AF state is the most stable one and lies 0.027 and $1.114 \mathrm{eV}$ lower than FM and NM states, respectively. Thus, the energy difference between $\mathrm{AF}$ and FM states per unit cell is $7 \mathrm{meV}$. This energy is small and comparable to that due to thermal fluctuation. Hence, we conclude that the FM and AF states are nearly degenerate. Their corresponding band structure and PDOS are plotted in Fig. 4. We see that FM state is half metallic, where the majority channel remains insulating with a band gap of $6.57 \mathrm{eV}$, and the minority channel is conducting. Analysis of PDOS shows that the magnetism on $\mathrm{N}$ atoms is mainly contributed by the unpaired $2 p_{z}$ orbital. This can be visualized from the isosurface of spin density in Fig. 4(e). The relevant mechanism is similar to what we discussed in $\mathrm{H}-\mathrm{BN}$ sheet. The binding of $\mathrm{F}$ atoms on $\mathrm{B}$ sites make the $2 p$ electrons on $\mathrm{N}$ sites unpaired. We also found that when two $\mathrm{F}$ atoms are introduced, two unoccupied energy bands are induced in the minority-spin channel just slightly above the valence band. When more $\mathrm{F}$ atoms are adsorbed, the system finally becomes half metallic.

For the AF state, the system becomes a direct band-gap semiconductor with a small band gap of $0.63 \mathrm{eV}$. Just above the valence band of pristine $\mathrm{BN}$ sheet, we note that there are four induced energy bands in the conduction manifold (two in majority and two in minority-spin channel). A large band gap exists above these two bands. The isosurface and twodimensional slice of spin density are plotted in Fig. 4(f). 


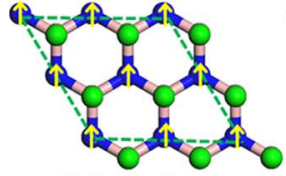

$\mathrm{FM}, \mathrm{m}=4 \mu_{\mathrm{B}}$ $\mathrm{E}=0.027 \mathrm{eV}$
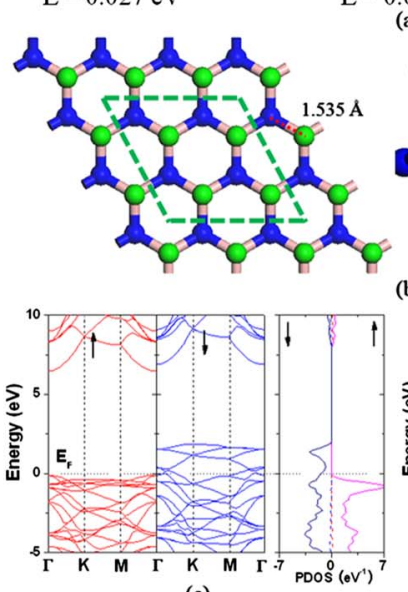

(c)

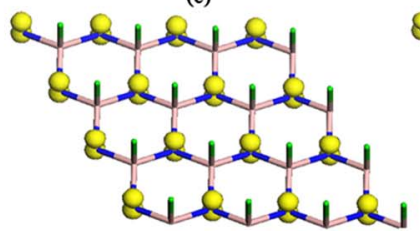

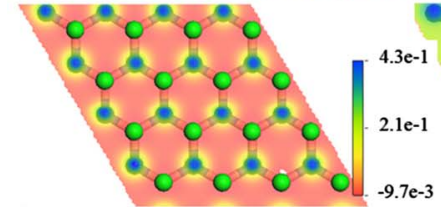

(e)

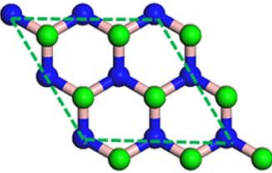

$\mathrm{NM}, \mathrm{m}=0 \mu_{\mathrm{B}}$ $\mathrm{E}=1.114 \mathrm{eV}$ (a)

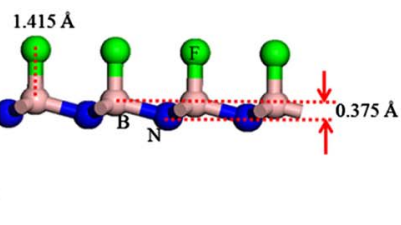

(b)

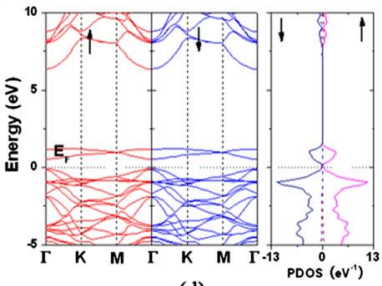

(d)

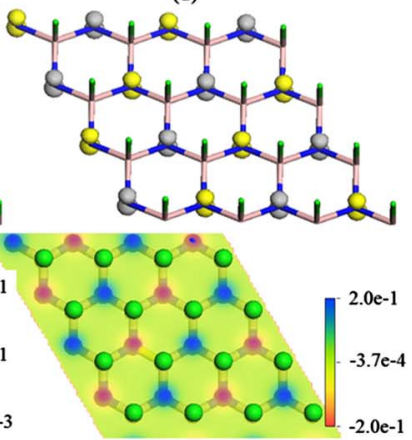

(f)
FIG. 4. (Color online) Calculated results of F-BN. (a) Relative energies for three magnetic coupling. (b) Structure of F-BN in AF state. [(c) and (e)] calculated band structure, PDOS, as well as isosurface $\left(0.5 e / \AA^{3}\right)$ and 2D slice of spin density of F-BN in FM state. (d) and (f) are same as (c) and (e) but for AF state.

\section{Applied stress on semifluorinated BN sheet}

We found that magnetic properties of fluorinated $\mathrm{BN}$ sheet are different from that in the fluorinated $\mathrm{BN}$ nanotube. ${ }^{28}$ The latter has been found to be FM. To see if this difference is due to strain caused by curvature effects on the $\mathrm{BN}$ nanotube, we applied in-plane tensile and compressive stresses in the fluorinated BN sheet. For the F-BN system under tensile stress, the energy difference between $\mathrm{AF}$ and FM states increases with higher tension and AF is always more stable than FM state. But for the compressive stress on F-BN, we found that the energy of FM state increases slower than $\mathrm{AF}$ state and at 5\% in-plane compression, FM state is energetically more stable than AF state by $32 \mathrm{meV}$ per unit cell (Fig. 5).

\section{E. BN sheet codecorated with $\mathbf{H}$ and $\mathbf{F}$}

As we have seen from above that hydrogenation of $\mathrm{BN}$ sheet is endothermic, but once the sheet is distorted, $\mathrm{H}$ can

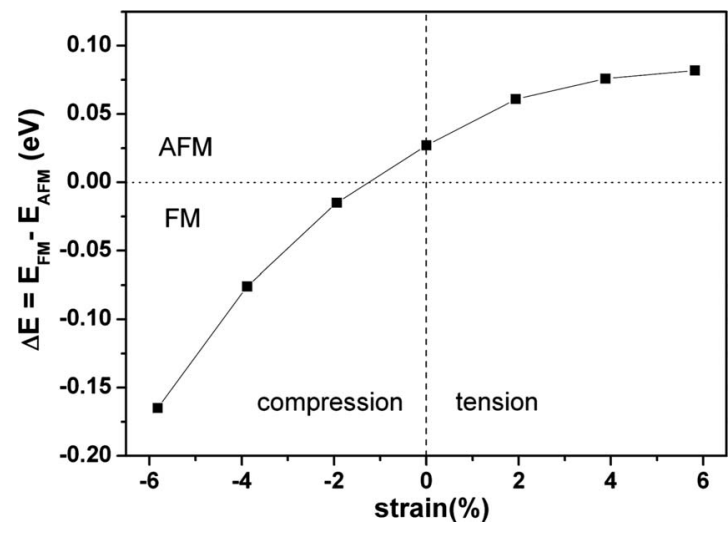

FIG. 5. Stability of magnetic state as a function of strain.

easily bind on B or $\mathrm{N}$ sites. Also, we find that fluorination is exothermic and can distort the sheet. Therefore, we can further introduce $\mathrm{H}$ to the semifluorinated $\mathrm{BN}$ sheet forming a codecorated sheet, where $\mathrm{F}$ atoms are attached to the $\mathrm{B}$ site while $\mathrm{H}$ atoms are attached to the $\mathrm{N}$ sites (labeled as F-BN-H, as shown in Fig. 6).

The optimized F-B and $\mathrm{H}-\mathrm{N}$ distances are shorter than those in F-BN and H-NB sheets indicating that the bonding is stronger than that in the systems discussed above. Conse-
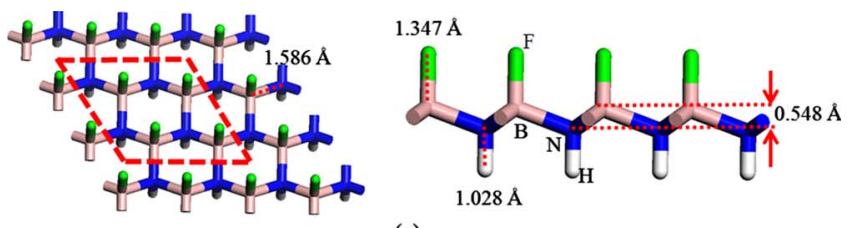

(a)

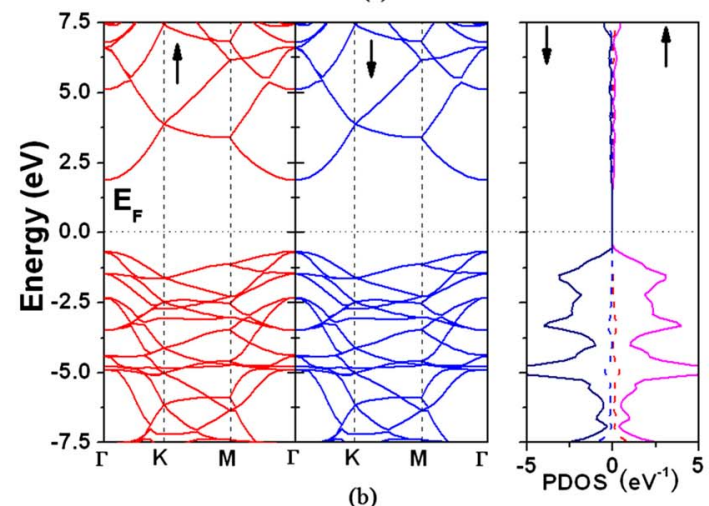

(b)

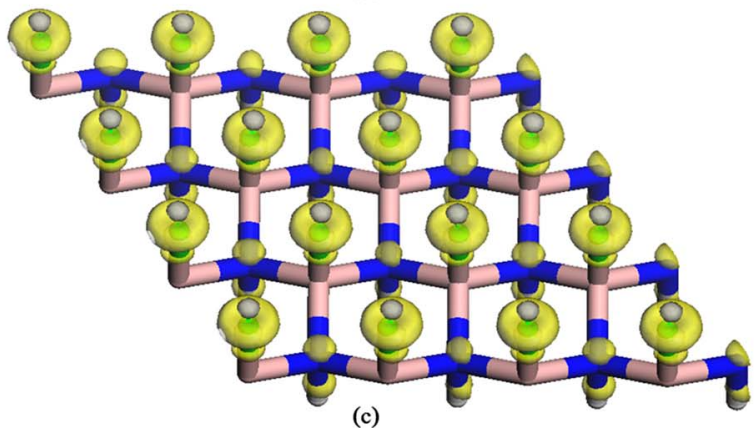

FIG. 6. (Color online) (a) Calculated geometric structure. (b) Band structure and PDOS of F-BN-H. (c) The charge-density difference $\left(\rho_{\mathrm{F}-\mathrm{BN}-\mathrm{H}}-\rho_{\mathrm{F}}-\rho_{\mathrm{BN}}-\rho_{\mathrm{H}}\right)$ of F-BN-H. 
TABLE I. Summary of electronic and magnetic properties of the systems studied. Symbol $s / h$ refers to semiconductor or half metal and $d / i$ refers to direct or indirect band gap of semiconductor. $E_{g}$ is the energy-band gap.

\begin{tabular}{lccccccc}
\hline \hline & $\mathrm{BN}$ & $\mathrm{H}-\mathrm{BN}-\mathrm{H}$ & $\mathrm{H}-\mathrm{BN}(\mathrm{FM})$ & $\mathrm{H}-\mathrm{NB}(\mathrm{AF})$ & F-BN (FM) & F-BN (AF) & F-BN-H \\
\hline$s / h$ & $s$ & $s$ & $h$ & $s$ & $h$ & $s$ & $s$ \\
$d / i$ & $d$ & $d$ & & $i$ & & $d$ & $d$ \\
$E_{g} / \mathrm{eV}$ & 4.71 & 3.33 & & 0.97 & & 0.63 & 2.55 \\
\hline \hline
\end{tabular}

quently, the distortion of the planar BN sheet is larger than that in the F-BN and H-NB systems as can be seen from the distance between $\mathrm{B}$ and $\mathrm{N}$ layers, which is calculated to be $0.548 \AA$ [Fig. 6(a)]. The codecorated F-BN-H system is a direct band-gap semiconductor with a band gap of $2.55 \mathrm{eV}$ which is smaller than those of pristine $\mathrm{BN}$, or $\mathrm{H}-\mathrm{BN}-\mathrm{H}$, but much larger than those in $\mathrm{H}-\mathrm{NB}(\mathrm{AF})$ and F-BN (AF) sheets.

What is more interesting is that codecoration using $\mathrm{H}$ and $\mathrm{F}$ atoms can induce anisotropy in the system. The pristine $\mathrm{BN}$ sheet is a two-dimensional isotropic system. When decorated with $\mathrm{F}$ atom on $\mathrm{B}$ sites and $\mathrm{H}$ atom on $\mathrm{N}$ sites, the large difference in the electron affinity of $\mathrm{H}$ and $\mathrm{F}$ makes the charge distribution asymmetric; $\mathrm{F}$ atoms receive electrons and become negatively charged on one side while $\mathrm{H}$ atoms donate electrons and become positively charged on the other side. This asymmetric charge distribution induced by the codecoration in $\mathrm{BN}$ sheet can be visualized from the electrondensity difference $\left(\rho_{\mathrm{F}-\mathrm{BN}-\mathrm{H}}-\rho_{\mathrm{F}}-\rho_{\mathrm{BN}}-\rho_{\mathrm{H}}\right)$ shown in Fig. $6(\mathrm{c})$. One can take advantage of this asymmetric charge distribution to promote self-assembly through charge interactions. Thus, the weakness of van der Waals interactions between the pristine $\mathrm{BN}$ sheets can be overcome and synthesis of more stable BN sheets by using linkers may be possible. One may wonder if the stacking of multiple F-BN-H sheets would only create $\mathrm{HF}$-intercalated $\mathrm{BN}$. We have found that the former is lower in energy than the latter by $0.27 \mathrm{eV} / \mathrm{HF}$, suggesting that stacking is stable against intercalation.

From above, we can see that the codecorated BN sheet with $\mathrm{F}$ and $\mathrm{H}$ on two sides becomes a two-dimensional Janus anisotropic structure. ${ }^{30}$ Janus anisotropy has attracted growing interest because of its applications in molecular recognition, self-assembly, photonic crystals, sensors, drug delivery, surfactants, solar cells, and display materials. We hope that similar to Janus particles, ${ }^{31,32}$ two-dimensional Janus structures of F-BN-H sheet may find potential applications.

\section{CONCLUSIONS}

We have systematically studied the modulation of electronic and magnetic properties through hydrogenation and/or fluorination of $\mathrm{BN}$ sheet. In Table I, we summarize the results. The main conclusions are as follows. (1) Hydrogenation is endothermic while fluorination is exothermic. (2) Our calculations show that fully decorated systems are all direct band-gap semiconductors. (3) In semidecorated systems, $\mathrm{H}-\mathrm{BN}, \mathrm{F}-\mathrm{BN}$, and $\mathrm{H}-\mathrm{NB}$, the unsaturated $\mathrm{N}$ or B atoms carry magnetic moments which are contributed mainly by $2 p_{z}$ orbital. H-BN and H-NB systems couple, respectively, ferromagnetically and antiferromagnetically. However, in F-BN system, the ferromagnetic and antiferromagnetic states are energetically nearly degenerate. (4) The induced energy band is located above the valence band or below the conduction band and reflects how electrons are transferred. For the $\mathrm{H}-\mathrm{BN}$ and F-BN in FM state, both the systems possess halfmetallic property with majority-spin channel being semiconducting while minority-spin channel being metallic. These are related to the extension of occupied and unoccupied energy bands above the valence band of pristine BN sheet. The $\mathrm{H}-\mathrm{NB}$ and F-BN systems in AF state behave as semiconductors with small energy gaps. (5) The in-plane strain can be used to tune the relative stability of FM and AF configurations of the F-BN system. (6) Codecoration with $\mathrm{H}$ and $\mathrm{F}$ atoms can introduce anisotropy in charge distribution, which can be used to facilitate self-assembly under an applied electric field, making it possible to overcome the weakness of van der Waals interactions between the pristine BN sheets. (7) All surface decorations of the $\mathrm{BN}$ sheet reduce the energy-band gap displaying different behaviors from that in the graphene sheet. The diverse properties of decorated $\mathrm{BN}$ sheets have the potential for wider applications of 2D-based materials and devices.

\section{ACKNOWLEDGMENTS}

This work is partially supported by grants from the National Natural Science Foundation of China (Grants No. NSFC-10744006, No. NSFC-10874007, and No. NSFC20973010), and from the U.S. Department of Energy.

\footnotetext{
*Corresponding author: sunqiang@pku.edu.cn

${ }^{1}$ K. S. Novoselov, A. K. Geim, S. V. Morozov, D. Jiang, Y. Zhang, S. V. Dubonos, I. V. Grigorieva, and A. A. Firsov, Science 306, 666 (2004).

${ }^{2}$ A. K. Geim, Science 324, 1530 (2009).
}

${ }^{3}$ W. Q. Han, L. Wu, Y. Zhu, K. Watanabe, and T. Taniguchi, Appl. Phys. Lett. 93, 223103 (2008).

${ }^{4}$ C. Zhi, Y. Bando, C. Tang, H. Kuwahara, and D. Golberg, Adv. Mater. 21, 2889 (2009).

${ }^{5}$ D. C. Elias, R. R. Nair, T. M. G. Mohiuddin, S. V. Morozov, P. 
Blake, M. P. Halsall, A. C. Ferrari, D. W. Boukhvalov, M. I. Katsnelson, A. K. Geim, and K. S. Novoselov, Science 323, 610 (2009).

${ }^{6}$ J. O. Sofo, A. S. Chaudhari, and G. D. Barber, Phys. Rev. B 75, 153401 (2007).

${ }^{7}$ J. Zhou, M. Wu, X. Zhou, and Q. Sun, Appl. Phys. Lett. 95, 103108 (2009).

${ }^{8}$ J. Zhou, Q. Wang, Q. Sun, X. S. Chen, Y. Kawazoe, and P. Jena, Nano Lett. 9, 3867 (2009).

${ }^{9}$ J. P. Perdew, K. Burke, and M. Ernzerhof, Phys. Rev. Lett. 77, 3865 (1996).

${ }^{10}$ G. Kresse and J. Furthmuller, Phys. Rev. B 54, 11169 (1996).

${ }^{11}$ G. Kresse and J. Joubert, Phys. Rev. B 59, 1758 (1999).

${ }^{12}$ H. J. Monkhorst and J. D. Pack, Phys. Rev. B 13, 5188 (1976).

${ }^{13}$ M. Topsakal, E. Akturk, and S. Ciraci, Phys. Rev. B 79, 115442 (2009).

${ }^{14}$ K. P. Huber and G. Herzberg, Constants of Diatomic Molecules (Van Nostrand Reinhold, New York, 1979).

${ }^{15}$ H. Xiang, J. Yang, J. G. Hou, and Q. Zhu, J. Am. Chem. Soc. 128, 2310 (2006)

${ }^{16}$ H. Cheng, X. Sha, L. Chen, A. C. Cooper, M.-L. Foo, G. C. Lau, W. H. Bailey III, and G. P. Pez, J. Am. Chem. Soc. 131, 17732 (2009).

${ }^{17}$ S. H. Lai, K. L. Chang, H. C. Shih, K. P. Huang, and P. Lin, Appl. Phys. Lett. 85, 6248 (2004).

${ }^{18}$ K. N. Kudin, H. F. Bettinger, and G. E. Scuseria, Phys. Rev. B 63, 045413 (2001).

${ }^{19}$ K. H. An, J. G. Heo, K. G. Jeon, D. J. Bae, C. Jo, C. W. Yang, C. Park, Y. H. Lee, Y. S. Lee, and Y. S. Chung, Appl. Phys. Lett. 80, 4235 (2002).
${ }^{20}$ I. S. Neretin, K. A. Lyssenko, M. Y. Antipin, Y. L. Slovokhotov, O. V. Boltalina, P. A. Troshin, A. Y. Lukonin, L. N. Sidorov, and R. Taylor, Angew. Chem., Int. Ed. 39, 3273 (2000).

${ }^{21}$ A. A. Popov, V. M. Senyavin, V. I. Keropanov, I. V. Goldt, A. M. Lebedev, V. G. Stankevich, K. A. Menshikov, N. Y. Svechnikov, O. V. Boltalina, I. E. Kareev, S. Kimura, O. Sidorova, K. Kanno, and I. Akimoto, Phys. Rev. B 79, 045413 (2009).

${ }^{22}$ Z. Slanina and F. Uhlik, Chem. Phys. Lett. 374, 100 (2003).

${ }^{23}$ A. A. Popov, V. M. Senyavin, O. V. Boltalina, K. Seppelt, J. Spandl, C. S. Feigerle, and R. N. Compton, J. Phys. Chem. A 110, 8645 (2006).

${ }^{24}$ L. G. Bulusheva, A. V. Okotrub, and O. V. Boltalina, J. Phys. Chem. A 103, 9921 (1999).

${ }^{25}$ S. I. Troyanov, P. A. Troshin, O. V. Boltalina, I. N. Ioffe, L. N. Sidonov, and E. Kemnitz, Angew. Chem. Int. Ed. 40, 2285 (2001).

${ }^{26}$ J. Jia, H. Wu, X. Xu, X. Zhang, and H. Jiao, J. Am. Chem. Soc. 130, 3985 (2008).

${ }^{27}$ P. A. Troshin, A. G. Avent, A. D. Darwish, N. Martsinovich, A. K. Abdul-Sada, J. M. Street, and R. Taylor, Science 309, 278 (2005).

${ }^{28}$ Z. Zhang and W. Guo, J. Am. Chem. Soc. 131, 6874 (2009).

${ }^{29}$ H. J. Xiang, J. Yang, J. G. Hou, and Q. Zhu, Appl. Phys. Lett. 87, 243113 (2005).

${ }^{30}$ P. G. de Gennes, Rev. Mod. Phys. 64, 645 (1992).

${ }^{31}$ Q. Sun, Q. Wang, P. Jena, and Y. Kawazoe, ACS Nano 2, 341 (2008).

${ }^{32}$ M. Wu, Q. Sun, Q. Wang, P. Jena, and Y. Kawazoe, J. Chem. Phys. 130, 184714 (2009). 